\title{
\$. 17.18.
}

\section{IISGHULANHA}

\section{I \\ CIIIIICA, FISICA E STORIA NATURALE \\ ANNO I. = PISA 1843.

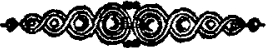

\section{SOMMARIO}

Sul freddo prodotto dalle correnti elettriche; Memoria del Prof. Luigi Pacinotli, pag. 237. - Estratto di una Memoria del Sig. $\boldsymbol{H}$. R. Goep. pert, sulla formazione degli strati lognosi nelle ceppaje d'abete, inserita negli Annali di Scienze Naturali di Parigi, pag. 272 - Lezioni Su i fenomeni Fisico-Chimici dei Corpi Viventi; Lezione VII del Prof. Carlo Matteucci, pag. 276.

\section{Sul freddo prodotto dalle correnti elettriche nei metall (1).}

\author{
MEMORIA DI LUIGI PACINOTTI \\ PROFESSORE NELL' I. E R. UNIVERSITA' DI PISA.
}

Sono ben note le interessanti sperienze che fino dal 1834 it signor Peltier pubblicò sull' effetto calorifico che la corrente elettrica produce nei metalli, allorquando gli percorre (2), ond' io, dovendomi da quelle dipartire, ne rammenterò solo quanto occorre perchè non sembri che voglia arrogarmi le altrui scoperte, e perchè possa comprendersi quel poco che io ho saputo aggiungere con alcuni miei stu-

(t) Questa memoria fu in parte inserita negli Annali di Fisica, Chimica e Matematiche del Sig. Majoccbi, ed è stata lelta alla Riunione di Torino (Vedi Annali, Tom. I. pag. 165); l'Autore peró l'ha accresciuta di altre sperienze, e di alcune di esse ha reso conto nzll' ultimo Congresso tenuto in Lucca.

(2) Bibl. Universclle, Tom. LVII, pag. 184.

N. 17. 


\section{8}

di sperimentali sul potere frigorifico delle correnti elettriche, dei quali mi propongo brevemente trattare. Non distinguerò, come fa il fisico francese, la corrente positiva dalla negativa, e mi atterrò piuttosto come è in uso fra noi al sistema franckliniano supponendo che una sola corrente abbia sempre luogo nel filo congiuntivo, e vada per questo dal polo positivo al negativo dell' apparato clettromotore .

Il signor Peltier stabili primieramente che l' elevazione di temperatura è uguale per tutta la lunghezza del metallo omogeneo percorso dalla corrente, menochè verso l' estremità sue ove si congiunge ad altri metalli. In secondo luogo, che nell' estremità del metallo la elevazione di temperatura si accresce allorchè la corrente elettrica passa da un peggiore in un miglior conduttore per l' elettrico, e diminuisce quando va dal migliore nel peggior conduttore. In terzo luogo stabili che vi sono alcuni metalli, e fra questi quelli che cristallizzano facilmente, nei quali invece di aversi minor riscaldamento si ha raffreddamento nel punto della loro congiunzione per effetto della stessa corrente. Aveva egli riscontrato questo fenomeno del raffreddamento nel bismuto, nell' antimonio e nel ferro oligisto, e per induzione suppose che lo avrebhe ottenuto nell' arsenico, ed essendogli mancato nel ferro dolce ed in altri metalli non cristallizzati concluse che fosse proprio di quelli che facilmente cristallizzano. Scmbrò dunque per le cose del signor Peltier che il freddo prodotto dalla corrente elettrica dovesse aversi per un fonomeno particolare ad alcuni corpi soltanto, o almeno dipendente dalla cristallizzazione; e sorprendente come esso aplariva per la sua novita fermò nel 1838 l'attenzione del Signor Lenz, il quale prendendo a confermarlo solo perì nelle copie composte di bismuto e antimonio, riusci a por fuori di dubbio (1) che allorquando la corrente voltaica è dirctta dal bisnuto all' antimonio, si ha raffreddamento nel luogo ove questi metalli si congiungono insieme.

Per un lato dietro questi fatti stabiliti dai chiarissimi fisici Peltier e Lenz era dimostrato che la corrente clettrica gode della proprietà di generar freddo nel passaggio dal bismuto all' antimonio; per l'altro il fenomeno sembrava troppo opposto all' effetto calorifico riconosciuto in tutti $i$

(1) Bibl, Cniverselle, T, XVII, pag, 387. 
casi nelle correnti. Talchè non mi pareva quello da attribuirsi alla cristallizzazione o alle azioni molecolari, e proprie soltanto dei corpi bismuto, antimonio e ferro oligisto, nei quali unicamente erasi riscontrato; e dubitai che troppa generalità si fosse accordata al poter calorifico delle correnti, e che bisognasse distinguere $i$ primi istanti in cui sono invasi i conduttori dall' elettrico dal tempo successivo, perchè gli uni manifestano il vero effetto dell' impouderabile nelle singole parti dei conduttori, e nell' altro il calore accresciuto in una parte si propaga nelle parti contigue ove prima di tal propagazione poteva pure esser raffreddamento. Mi posi pertanto a studiare con accuratezza il fenomeno del freddo prodotto dalla corrente elettrica, e mi risultò meno singolare, e più importante di quello che mi era sembrato sulle prime. Non intendo qui riferire tutti i particolari della lunga serie d'esperienze che ho eseguite su questo soggetto, ma solo di annunziare i fatti più interessánti che ho potuti constatare.

\section{PARTE PRIMA}

Studio del fenomeno del frcddo prodotto dalla corrente elettrica in alcuni conduttori metallici.

Ripetuti gli esperimenti dei signori Peltier e Lenz con apparati analoghi a quelli dai medesimi usati, riconobbi per mezzo del galvanometro e del termometro ad aria che allorquando la corrente di un grand' elemento voltaico passa dal bismuto all' antimonio si eccita nella loro saldatura il raffreddamento, che massimo si manifesta su' primi istanli e dopo diminnisce, e si converte in un piccolo riscaldamento. Con qualche particolar diligenza notato l' andamento di queste variazioni di temperature, ho riconosciuto che mandando la corrente dal bismuto all'antimonio l'elevazione di temperatura, che si ottiene dopo il freddo, non raggiunge mai quella che viene eccitata dalla corrente volta in verso contrario, finchè quella corrente esiste; estinta poi ch' ella sia il riscaldamento si aumenta presso a poco. al grado che si avrebbe col mandar la corrente dall' antimonio al bismuto. Questo aumento dimostra in un modo manifesto un poter refrigerante proprio della corrente, il quale non è transitorio, ma si mantiene finchè seguita quella; perchè il riscaldamento che succede dopo i primi 
istanti è dovuto alla propagazione del calorico, e più grande si farebbe se non lo impedisse il poter refrigerante della corrente, come infatti accade allorchè questa cessa di esistere .

Provato cosi che il poter refrigerante della corrente non e un effetto istantaneo prodotto dal suo primo passaggio, come potea supporsi per l' esperienze dei citati fisici, mi rivolsi a ricercare se era questo un fenomeno proprio di alcuni metalli solamente. Aveva io già appreso dalle sperienze fatte colle coppie di bismuto e antimonio di non dovermi fermare ad osservare nella congiunzione dei metalli percorsi dall' elettrico lo stato permanente di temperatura, ma quello che vi si produce nel primo istante, e quello che vi ha luogo cessata la corrente. E con tale scorta posi sotto esperimento, oltre al bismuto e antimonio, il ferro, lo zinco, l'ottone, il rame, lo stagno e l' argento, combinando fra di loro questi metalli in tutti i modi possibili e dopo avere in diverse maniere variato l'esperimento', ebbi luogo di assicurarmi che in tutte le coppie si riproduce il fenomeno del freddo, perchè la saldatura dei due metalli si scaldò quando la corrente andava in una direzione e si raffreddó più o meno secondo la natura dei metalli quando la volgevo nella direzione opposta . Data percio la conveniente direzione ad una corrente voltaica, allorchè passa da uno in un altro metallo, essa genera un raffreddamento nel luogo ove sono saldati $i$ due melalli con differente intensità secondo la natura dei metalli slessi. $\mathbf{E}$ il medesimo ottenni, tanto sperimentando sopra strisce metalliche saldate insieme, quanto sopra fili di metallo egualmente saldati : se non che più comodo mi riesciva sperimentare colle prime. Ad ottenere il fenomeno del freddo dovetti dirigere la corrente dal bismuto all' antimonio, dal rame al ferro, dallo zinco al ferro, dall' ottone al ferro dal rame allo zinco, dal rame all' argento, ec. ; che se io dirigeva la corrente in verso opposto la saldatura dei metalli mi si scaldava notabilissimamente. Per stromento termoscopico in quest' esperienza usai una coppia termo-elettrica unita al galvanometro, e devo la nozione del fatto alla prontezza con cui si muove l'ago nello strumento. Per questa possono conoscersi tutte le alterazioni di temperatura che seguono nei successivi istanti : così il raffreddamento mi era indicato: $10^{\circ}$ dall' andamento dell' ago del gatvapometro nel suo primo movimento, il quale si faceva in 
verso opposto a quello che poi ne succedeva per il riscald ${ }^{*}$ mento ; $2 .^{\circ}$ dal non essere il successivo riscaldamento tanto grande quanto quello che si sarebbe avuto mandando la corrente voltaica in direzione opposta; $3 .^{\circ}$ dall' ottenere un aumento di temperatura dopo avere estinta la corrente. Anche Peltier aveva avvertito il secondo di questi fatti, ma per la mancanza degli altri non potè rilevare, che quando la corrente è convenientemente diretta da uno in un altro metallo, si ha nella loro saldatura produzione di freddo, e dovette limitarsi a dire, come l'esperienza gli accennava, che si ha minor riscaldamento col mandar la corrente da uno in un altro metallo in una direzione, e maggiore in opposta. Mancando il primo resultato, che direttamente mostra il raffreddamento, e l'ultimo che fa conoscere esser permanente il poter refrigerante della corrente conveniva concludero come il Fisico francese; ma conoscendo questi due fatti ben si scorge come si abbia a intendere il secondo. Questo mostra un aumento di temperatura anche nel luogo ove si congiungono i due metalli non per effetto diretto della corrente, ma perchè quella essendo stabilita, il calorico che si c accumulato nelle parti circonvicine, si propaga sulla saldatura .

ll mio modo di sperimentare mi sembra preciso, e molto conveniente allo scopo: stava in comunicazione con un buon galvanometro (ed era precisamente quello dell'apparato del Melloni) un elemento termo-elettrico di bismuto e antimonio; su questo posava coll' intermedio di una carta la saldatura delle due striscie metalliche che dovevano mostrare il raffreddamento, e vi era aggravata con un peso affinchè meglio fosse stabilito il contatto; due fili di rame ponevano in comunicazione le due strisce con due tazzette piene di mercurio ; ed in quelle erano portati i reofori di un grande elemento voltaico a spirale. Quindi facilmente si faceva passare la corrente nella coppia delle strisce, 0 ' interrompeva il suo passaggio, o s' invertiva ; perchè a ciò bastava immergere nel mercurio o tirar fuori i due reofori, o invertire la loro posizione nelle tazzette. Quando la corrente aveva ottenuta la direzione adattata, accadeva un subitaneo raffreddamento nella saldatura delle strisce metalliche, il quale si comunicara all' elemento termo-elet. trico, e l'ago del galvanometro marciava verso il freddo. Un momento dopo si elevava la temperatura anche nella saldatura delle strisce metalliche, ed io credo che ciò, co* 
me ho detto, avvenisse per calorico condotto dalle parti circonvicine delle strisce stesse, scaldate fin dal primo istante per effetto della corrente; e l' ago del galvanometro tornava a zero, e deviava dalla parte opposta verso il caldo, e dopo un certo tempo, cessate le oscillazioni, acquistava una posizione stabile. Levati allora i reofori dalle tazzette, e interrotta cosi la corrente, si aumentava la deviazione dell' ago, ed accennavasi elevazione di temperatura presso a poco eguale a quella che si sarebbe ottenuta dando alla corrente direzione opposta. Questi fatti, come, ognun vede, non lasciano dubbio sulla generazione del freddo in tutte le coppie metalliche per effetto della corrente. Molte altre indicazioni interessanti io scorgeva pel diverso modo tenuto dall' ago nell' avanzarsi secondo la diversa direzione della corrente, le quali mi avvisavano la legge con cui procede il riscaldamento, ma di questo non è qui luogo a parlare. Ho ritrovato che un tal metodo di esperimentare è comodissimo quando l'acqua della pila è assai acidulata, ma quando ha poco acido convien portare i reofori direttamente a contatto colle strisce metalliche per aver sensibile il raffreddamento .

Passando ora alla ricerca del legame che presenta il fenomeno del raffreddamento con gli altri già noti nella scienza, ci ricorderemo, come ho detto di sopra, che il signor Peltier stabilì essere alla differenza di conducibilità dei metalli dovuto il maggiore o minore riscaldamento che si ottiene nella loro saldatura. E poichè quello che egli giudicava fenomeno di minor riscaldamento, dopo le cose precedenti, deve aversi per reale raffreddamento, può domandarsi se quando la corrente va dal metallo più conduttore in quello meno conduttore si abbia sempre raffreddamento. A me non sembra che sia così : giacchè non (1) credo che l' antimonio sia meno conduttore del bismuto, nè l' argento lo sia meno del rame, e troppe altre eccezioni dovrebbero farsi alla regola. Osservo poi che la sola differenza di conducibilità non può essere la cagione del maggior riscaldamento o del raffreddamento, perchè il fenomeno si effettua al maggior grado in una coppia di bismuto e antimonio sebbene

(1) Alcune esperienze da me fatte dimostrano l' Antimonio tre o quattro volte più condultore del Bismuto, ed II Signor Lenz, rappresentando con 100 la conducibilità del Rame, trova che quella dell' Antimenio é 8,87, c quella del Bismuto 2,58. Bibl. Univers. ottobre 1830. 
piccola sia la differenza della loro conducibilita, ed il medesimo posso ripetere per una coppia di ferro e stagno: forse vi contribuisce anche la gravità specifica, e la capacità per il calorico. Non proponendomi di discutere ciò, mi limito a quello chc vien provato dall' cspcrienze. Queste dinostrano che un legame manifesto esiste tra il fenomeno della produzione del freddo, per effetto della corrente elettrica e quello della produzione della corrente per effetto del caloricu; e tutte mi hanno provato che la correnle voltaica produce freddo, quando in una coppia metallica è mandata in quella dircsione in cui suole eccitarsi la corrente termo-elettrica, allorchi si scalda la congiunzios ne dei due melalli. Qucsto teorema in seguito forse apparirà fecondissimo, esscndo del tutto ignoto il legame che esiste tra la produzione della corrente termo-elettrica, e gli altri fenomeni clettro-dinamici. 'n' cecezione singolare mi era stata presentata da una coppia di argento c rame, perchè in essa aveva ritrovato diretta dall' argento al rame, la corrente termo-elettrica, che producesi collo scaldarc la congiunzione dei due metalli, ed il rreddo in questa lo aveva ottenuto, quindo vi introdussi una corrente diretta dal rame all' argento. Ma avendo moltissime volte ripeluto questo esperimento, e con diversa intunsití di corrente voltaica, ho in alcuni casi ritrovato il fenomeno del fredilo anche quando la corrente è diretta dall' argento al rame. Quel. lo poi che mi ha tolta l'eccezione ì slato l'aver veduto che per un forte riscaldamento prodotto con un lume ad alcool nclla saldatura, la corrente termo-elettrica va dal rame all' argento . Anzi ho avuto così una doppia conferma del tcorema, poichè la corrente termo-elettrica andando nei due versi nella coppia rame c argento, secondo la intensità del riscaldamento, anclic la corrente voltaica secondo la sua intensità produce il fenomeno del freddo in am. bedue le opposte direzioni. Non per la via dell' esperienza ma col semplice ragionamento si poteva venire alla conclusione che il fenomeno del freddo fosse legato col termoelettricismo e lo aveva fatto il Poggendorff fino dal 1838 non prima che io escguissi le precedenti csperienze, ma prima però che le facessi pubblichc col parlarne al Congresso di Torino. Bramando che gli Scienziati abbiano buon grado al Poggendorff di qucll' oltima idca, parmi che essa won avrebbe acquistalo il conveniente grado di interesse se prima non si fosse dimostrato che in tutte le coppie me- 
talliche può ottenersi il fenomeno del freddo, e coll' intensità che dirò in appresso .

L'azione, voglio dire, producendo una reazione poteva ben prevedersi che in quei casi nei quali lo sbilancio di temperalura produce una corrente elettrica, dovesse reciprocamente la corrente dare uno speciale sbilancio di temperatura. Ed accennava questo principio il vedersi generare il freddo più facilmente nelle coppie di bismuto e antimonio, che in quelle dl altri metalli, essendo le prime le più atte per il termo-elettricismo. Poteva anche prevedersi che quello special sbilancio di temperatura fosse di riscaldamento quando si manda la corrente in una direzione, e di raffreddamento quando si fa andare in direzione opposta, perchè il riscaldare la congiunzione di due metalli eccita la corrente elettrica diretta in un senso, e il raffreddarla la eccila in senso contrario. Ma per qual ragione deve aversi il freddo quando la corrente va nella direzione in cui suole nuoversi quando si scalda la congiunzione dei due metalli, piuttosto che in quella opposta che prende quando si raffredda quella lor conginnzione? Sarebbe questa forse la via per dimostrare che il calorico e l' elettrico dipendono ambeduc dall' ctere? L' etcre che si muove di molo progressivo compone la corrente clettrica : questo ha da agire in modo analogo ai liquidi : la corrente di acqua che urta contro l'aria o contro l' alveo ne fa oscillare le particelle, e tutta oscilla la massa acquea per cui s' increspa la sua superficie: lo stesso moto oscillalorio si farà nclle particelle ponderabili del corpo che conduce la corrente elettrica e nclle particelle stesse dell' ctere che sta dentro a quel corpo, ed in cio consistera l' elevazione di temperatura che generalmente si produce nci metalli dal passaggio della corrente clettrica . Quando la corrente passa da uno in un' altro metallo sari come quando una corrente di acrua passa da un recipiente in un' altro, da un alveo renoso ad uno ghiajoso o viceversa: Han luogo in quel passaggio dei vortici, dei rigurgiti, e in generale un alterazione dei moti oscillatorj nella massa acquea : si dcvono generar dunque speciali sbilanci di temperatura quando la corrente elettrica passa da un metallo in un' altro. Or sarebbe a determinarsi in quali casi si vedono più agitate da moti oscillatorj le masse acquee, ed in quali si vedono scorrere più placide allorchè passano da uno in un altro recipiente : questo ci darebbe lume per conoscere qua- 
le sia la proprietà dei due metalli bismuto ed antimonio che fa generar distruzione di moti oscillatori quando va la corrente elettrica dal primo nel secondo metallo e che li aumenta quando va dal secondo al primo. Anche lo studio delle costituzioni atomistiche dei corpi dai quali esce la corrente a confronto di quelle dei corpi nei quali entra quando si ha il fenomeno del freddo, ci porterebbero a scoprire dei dati interessantissimi per queste dottrine.

Onde meglio stabilire il fatto fondamentale della relazione tra il fenomeno del freddo ed il termo-elettricismo convien determinare se esista proporzionalità tra la corrente termo-elettrica e l'intensita, colla quale si produce il raffreddamento. Per provare se ciò avesse luogo istituii delle sperienze di confronto, e ne ottenni i resultati che si possono vedere nella seguente tavola.

\begin{tabular}{|c|c|c|c|c|}
\hline $\begin{array}{l}\text { Direzione della corrente } \\
\text { termo-elettrica e della } \\
\text { corrente voltaica che pro- } \\
\text { duce il freddo. }\end{array}$ & 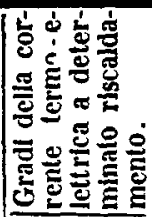 & 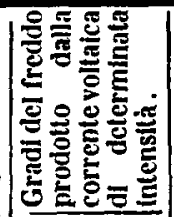 & 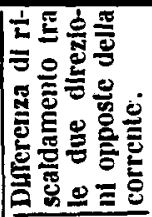 & 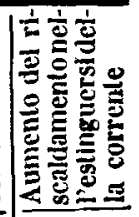 \\
\hline $\begin{array}{l}\text { Dal bismuto all'antimonio } \\
\text { Dallo stagno al ferro. . . } \\
\text { Dal rame al ferro . . . } \\
\text { Dallo zinco al ferro . . } \\
\text { Dall' ottone al ferro . . } \\
\text { Dal rame allo zinco . . } \\
\text { Dal rame all' argento . } \\
\text { Dall'argento al rame . }\end{array}$ & $\begin{array}{r}800 \\
41 \\
40 \\
39 \\
39 \\
6 \\
-\quad 5 \\
5\end{array}$ & $\begin{array}{r}16^{0} \\
4 \\
3,5 \\
3 \\
2,5 \\
0,5 \\
1 \\
0,3\end{array}$ & $\begin{array}{r}21^{0} \\
6 \\
10 \\
10 \\
7 \\
4 \\
3 \\
-\quad 3\end{array}$ & $\begin{array}{r}20^{0} \\
4 \\
16 \\
3 \\
2,5 \\
0,5 \\
1 \\
9,3\end{array}$ \\
\hline
\end{tabular}

In questa ponendo attenzione ai numeri della prima co. lonna ed a quelli respettivi della seconda, non si scorge una perfetta proporzionalità tra la intensità della corrente termo-elettrica, e quella che appartiene al fenomeno del freddo prodotto dalla corrente voltaica, pure ben si vede che in quelle coppie metalliche ove la elettromotricita per il calorico è maggiore, si ha anche maggiore l' effetto frigorifico della corrente elettrica, e presso a poco vi è proporzionalità frà questi due fenomeni. Dalla terza e quarta colonna, oltre e questa relazione, rilevasi che i numeri rappresentanti la differenza di temperatura che acquista permanentemente la saldatura metallica, allorchè si manda la corrente nelle due opposte direzioni, sono poco diversi da 
266

quelli che indicano l'aumento di temperatura dopo che è stata estinta la corrente. Che se inoltre avvertiamo qualche cosa doversi attribuire ai piccoli errori inevitabili sempre in esperienze si delicate, potremo concludere che esiste un rapporto diretto fra $i$ due menzionati fenomeni.

Piacque ad alcuni chiarissimi Scicnziati suggerirmi che convenirat esperimentare su metalli tenuti in differenti mezzi, e variare la saldatura, o il modo di congiungerli; e per soddisfare a si giusti riflessi come anche ad altre mie idee sulla intensità della corrente che produce il freddo, ripresi lo studio del soggetto. Sperimentai sopra una coppia di bismuto e di antimonio, collocate nel vuoto boileiano e nel gas idrogene, e posso dire di avere ottenuti i resultati che aveva avuti nell' aria non contando certe piccole differenze da attribuirsi alla difficoltà che proveniva dalla maggior complicanza dell' apparato. Parimente posi la coppia metallica nell' acqua, e nell'olio d'oliva, cioe in un mezzo per l'clettrico assai buon condutlore, ed in uno coibente, la copersi poi anche di uno strato di cera ed ebbi dal galvanometro sempre la prima deviazione che indicava il freddo presso a poco allo stesso grado che l' aveva ottenuta nell' aria, ed anzi più persistente, e debolissime mi comparvero le altre due deviazioni, quella ciò̀ del riscaldamento della saldatura, quando la corrente idroelettrica percorre da un certo tempo i metalli, e l'altra dell'aumcnto di questo riscaldamento, quando la corrente cessa di esistere. Ben si vede che tali differenze dipendono dal magrgiore assorbimento di calorico che si fa dal mezzo, onde può stabilirsi essere indipendente il fenomeno del freddo dal mezzo, nel quale è collocalo il melallo; e lo stesso mi è scmbrato potersi concludere per l'opposto fenomeno del riscaldamento prodotto dalla corrente. Per studiare l'azione della saldatura ho eseguite non molte esperienze, ma tali da pormi fuori di dubbio che il fenomeno si produce egualmente con metalli posti a contatto o solo con incastro forzato, o con la fusione di alcune loro parti, o con saldatura ad argento, o con saldatura a stagno. Lbbi anche luogo d'osservare che l'estensione per la quale comunicano fra loro i due metalli non deve essere tanto piccola onde sia assai intenso il raffreddamento.

Relativamente alla natura ed all' intensità della corrente, avverto essermi mancato il fenomeno mentre agiva con scariche anche forti di una o più bocce di Leida, come 
pure con le correnti termo-elettriche e con quelle magneto-elettriche. Queste correnti avevano peri debolissima forza, in confronto di quclla idro-elettrica che io soleva adoperare, al che dirò doversi attribuire la mancanza del fenomeno. Infatti non l'ottenni neppure da una corrente idro-elettrica che al galvanometro mostrava la stessa forza delle precedenti. Ed avendo poi per gradi accresciuta l'intensità della corrente idro-elettrica, vidi che solo mi si manifestava qualche debol segno di freddo sulla coppia bismuto e antimonio, quando usai un clemento elcttromotore di rame e zinco di circa sei pollici quadrati di superfice immerso in acqua mediocremente acidulata con acido solforico . Variando l' acqua acida potei corrispondentemente variare la superficie dell' elcmento voltaico, ed avere la stessa intensità di corrente al galvanometro;ed anche mantenni questa stessa intensità adoprando pile di un differente numero di elementi ; così sperimentai con la stessa intensità in differenti pile, e con intensità differente nella stessa pila, e giunsi a stabilire: $1 .^{\circ}$ ogni qualvolla torna la corrente voltaica alla stcssa intensilà, qualunque sia il liquido e il numero degli elementi, si ha il fenomeno del freddo ed anche quello del riscaldamento allo stesso grado: 2.0 al variar del$l$ 'intensità galvanometrica della corrente, proporzionalmente, presso a poco, varia l' intensità del raffreddamento e respettivamente anche del riscaldamento. Non ho potuto estendere l' esperienze a correnti di una grandissima forza, ma è credibile che anche queste diano un proporzionato abbassamento di temperatura, onde il fenomeno del freddo potrà con quelle esser meglio studiato. Io frattanto son contento di aver mostrato come eccitato il moto dell' elettrico in un conduttore metallico, questo in certi punti viene scaldato ed in altri raffreddato con forza proporzionale alla intensità magnetica della corrente, e con quella stessa legge colla quale raffreddandolo e scaldandolo in quegli stessi punti si porrebbe l' elettrico in corrente.

\section{P ARTE SECONDA}

Della corrente secondaria prodotta nei conduttori fatti a pezis alternati di due differenti metalli.

Per dire di alcuni fatti che mi sono stati discoperti dalla trovata analogia tra l' effetto frigorifico delle correnti elet- 


\section{8}

triche e la termo-elettromotricità, mostrerò come possa tal* volta una corrente voltaica, incanalata in certi conduttori, eccitarvi un' elettromotricità contraria, a somiglianza di quella che il Ritter scoperse nella sua pila, ed il Marianini ed altri in alcuni fili metallici. Supposto che non si stabilisca equilibrio di temperature in tutte le parti del metallo subito dopo che è interrotta la corrente, riman facile dimostrare che un conduttore costruito con pezzi di due differenti metalli posti alternativamente, come sono le pile termo-elettriche, deve acquistare un elettromotricità propria cessala la corrente che lo avrà percorso, e deve manifestare una corrente volta in verso contrario alla primiliva. Infatti si faccia percorrere da una corrente voltaica una pila di bismuto, e antimonio, in modo che essa entri per il polo bismulo, si raffredderanno le saldature $1,3,5$, 7 , ec. ove la corrente passa dal bismuto all' antimonio, e si riscalderanno le altre $2,4,6,8$, ec. nelle quali la corrente riman diretta dall' antimonio al bismuto; per conseguenza appena cessata la corrente voltaica se l'equilibrio di temperatura non si ristabilisce perfettamente, facendo comunicare col galvanometro i poli della pila termo-elettrica, vi si scorgerà una corrente che esce dal polo bismuto, ed è perciò contraria alla voltaica primitiva. L' esperienza mi ha confermato tuttociò nelle pile di bismuto e di antimonio, ed in quelle di stagno e ferro, ed in altri apparati che imitano la costruzione delle pile termo-elettriche, come sarebbe un filo di ferro frapposto a due di rame. Onde ho dovuto riconoscere la verità dclla induzione fatta col ragionamento, e ritencre che esiste nell' effetto frigorifico delle correnti un nuovo modo di svolgere elettromotricità in alcuni corpi.

Abbiasi dunque per stabilito che se un conduttore formato a pezzi alternativi di due metalli, come sono le pile termo-elettriche vien fatto percorrere da una corrente elettrica, e se poi cessando di farvi passar quella, si pongono le sue estremità in comunicazione coi fili del Galvanometro, trovasi che il conduttore è percorso da un altra corrente in direzione opposta a qualla che aveva la prima. Io chiamo quest'ultima corrente primaria, e l'altra secondaria, l'origine della quale è manifestamente l'azione di freddo e di caldo che nelle alternative saldature dei due metalli ha prodotta la corrente primaria. E chi volesse assicurarsi che la corrente secondaria è l'indizio della produzione del freddo agirà nel seguente modo. Dopo di 
aver mandata nel conduttore la corrente primaria in una direzione, e dopo di avere esplorata la corrente secondaria che vi si genera, lo che si fa in pochissimi minuti second, subito si mandi in quello stesso conduttore la corrente primaria in direzione opposta, e si torni ad esplorare la nuova corrente secondaria. Si troverà, che anche questa è opposta alla sua primaria, e perciò opposta all'altra secondaria che si era avanti generata. Dunque coll'invertire la corrente primaria si sono scaldale le saldature che dianzi si erano raffreddate, e si sono raffreddate quelle che prima eransi fatte calde. Questo toglie il dubbio che la corrente secondaria provenisse dall'essersi alternativamente le saldature scaldate una meno e l'altra più, e mostra che alcune non soffrono elevazione di temperatura minore delle altre, ma un reale abbassamento di temperatura. Infatti se la corrente secondaria fosse effetto di minor riscaldamento, e non di rafireddamento in alcune saldature, quando invertita la corrente primaria si scaldano di più quelle saldature che prima si erano scaldate di meno, e si scaldano di meno quelle che prima si erano scaldate di piü, si stabilirebbe un'uniformità di temperatura in tutte le saldature, e non comparirebbe l'altra corrente secondaria opposta alla prima, e presso a poco intensa quanto quella. Onde può dirsi che la produzione della corrente secondaria corrisponde alla produzione del freddo. Questo ci porta a riconoscere che la corrente secondaria $\dot{e}$ un metodo di studiare ed esplorare il freddo prodotto da una corrente elettrica, e come tal metodo mi sia riescito efficace resulterà da quello che sono per dire.

Ho detto di sopra che usando il primo metodo di sperimentare non potei oftenere indizio di fredda dalle correnti termo-elettriche, nè da quelle magneto-elettriche, nè dalle scariche della boccia di Leida. Aveva ottenuta una deviazione appena sensibile nell'ago del Galvanometro per il freddo prodatto nel luogo ove si congiungono un pezzo di bismuto con un pezzo d'antimonio, quando io vi facevá passare una corrente generata da un'elemento voltaica rame $e$ zinco di sei pollici in superficie, fatto agire in acqua pura; ed inoltre aveva determinato essere almeno entro a certi limiti il fenomeno del freddo proporzionale alla forza magneto-elettrica della corrente . Per questo conclusi che le correnti stero-elettriche da me poste sotto l'espepimento non potevano dare sensibile indizio del freddo che 
il loro passaggio nei conduttori metallici avesse potuto produrre. D'altra parte il fenomeno ofre un tale interesse che merita essere studiato, con qual legge si produce anche dalle debolissime correnti idro-elettriche, e se le correnti elettriche di diversa natura lo producono in egual modo, e tutte nell'unione dei due metalli, quando vi sono mandate in quel verso nel quale va la corrente termo-elettrica che si ottiene scaldando il luogo stesso di congiunzione dei due metalli. Quella corrente idro-elettrica che ho sopra rammentata dovuta ad un elemento voltaico rame e zinco di sei pollici in superficie fatto agire in acqua pu$\mathrm{ra}$, fu da me incanalata in una pila termo-elettrica di dodici elementi bismuto e antimonio e vi generó una corrente secondaria di 28.0 Ognun vede che una simil deviazione data all'ago del galvanometro è ben vistosa, e che percio la corrente secondaria offre un mudo attissimo per dimostrare in una pubblica lezione il fenomeno del freddo anche con correnti molto deboli. E poichè fra le tante macchine interessantissime di cui si è arricchito il Gabinetto di fisica dell'Universita di Pisa per cura del mio chiarissimo Collega prof. Matteucei vi era l' apparato immaginato da Pelter per mostrare il fenomeno del freddo; mi venne vaghezza di confrontare l' uso della corrente secondaria colla squisitezza di questo. L'apparato Peltier ottimamente inteso per provare il freddo che si produce nella saldatura di un pezzo di bismuto con un pezzo di antimonio, ̀̀ composto di due parti distinte. Nella prima si raccoglie il freddo con due elementi termo-elettrici bismuto e antimonio che toccano uno per di sopra e l'altro per di sotto la saldatura nella quale è mandata la corrente, e comunicano col galvanometro. Nella seconda questa saldatura sta entro ai globi di un termoscopio differenziale ad aria e questo fluido condensandosi al freddo che vi si produce, l'indice del termoscopio mostra il fenomeno col suo movimento; se non si usa un globo solo ma ambedue, convien riscaldare la saldatura in uno, e raffreddar quella nell'altro e l'indice mostra cumulati gli effetti del freddo e del caldo. Lascerò la descrizione di molte particularità che non interessano al mio discorso ; e avvertirò che sebben tal macchina sia in tutte le parti disposta con somma intelligenza, pure introdottavi la corrente idro-elettrica da me sopra menzionata quando usai la seconda parte dell'apparato con un sol glodo, il movimento dell' indice termoscopico fu insensibile; 
e quando adoprai la prima parte ebbi al galvanometro una deviazione di soli cinque gradi. Il resultato di cinque gradi in confronto di quello di $28 .^{\circ}$ che aveva ottenuti coll' uso della corrente secondaria, fa comprendere la superiorita di questa in confronto cogli altri mezzi fin'ora usati per dimostrare il fenomeno del freddo. Superiorità che comprendesi a prima vista solo si consideri, che il conduttore col numero delle sue alternative dei due metalli forma un moltiplicatore, e che la variazione di temperatura non si ha da comunicare da corpo a corpo, ma deve agire nel corpo stesso ove si ritrova. Merita attenzione anche la semplicità dei pezzi che possono usarsi per mostrare il fenomeno del freddo; un qualsivoglia piccol pezzo di rame e di zinco può dare la corrente primaria, e la corrente secondaria può benissimo esser prodotta in una catenella a maglie alternate di ferro c di rame.

Istruito così sulla efficacia della corrente secondaria, doveva venirmi in mente di provar questa per dimostrare il fenomeno del freddo prodotto delle correnti stero-clettriche, cioè dalle termo-elettriche e da quelle magneto-elettriche. lina pila a dugento elementi di bismuto e antimonio riscaldata mi diede la corrente termo-clettrica che usata, come primaria, la introdussi in una pila a dodici elementi bismuto e antimonio. In quest' ultima ottenni una corrente sccondaria di quattro gradi del galvanometro. Invertita la corrente primaria si invertiva la secondaria, onde chiarissimamente era provato che la corrente termo-elettrica produce colla legge delle idro-elettriche il fenomeno del freddo e quello del caldo nei conduttori melallici. Non avrei a dir vero creduto di poter giungere a questa prova prima di conoscere l'efficacia della corrente secondaria : e che io sappia nessun fisico ha provato non solo il fenomeno del freddo ma neppur quello del caldo prodotto dalle correnti lermo-elettriche. Col ragionamento si prevedevano: pur non lascia di far sorpresa l'osservare che una debule corrente termo-elettrica riscalda e ralfredda in modo chiaramente visibile un con-duttore metallico di piü linee di sezione. Per le correnti magneto-elettriche, usai una semplice calamita non molto forte armata alla Nobili ponendo l' elica della sua ancora in comunicazione con una pila di dodici elementi bismuto e antimonio, e vi ottenni nell' attaccare o distaccare l'ancora una corrente secondaria di 3 gradi del galvanometro. Ed è piacevol cosa vedere come una corrcnte si fugace qua- 
le è quella che si ha nello staccare o attaccare l'ancora puo cagionare il fenomeno del freddo e sbilanci assai permanenti nella temperatura delle diverse parti del conduttore metallico; sperava che la scarica della bottiglia riguardata come una corrente molto fugace, volesse imitare la corrente della calamita elettrica, ma per ora le prove non mi han corrisposto.

Prof. Luigi Pacinotti

estatito di una mbioria del sig. H, R. Gobtpert

SULLA FORMAZIONE

degli stratl legnosi nelle ceppaje d' Abete,

INSERITA

Neglo aNNALI DI SCIENzE NATURALI DI PARIGI

Glà da molto tempo conoscesi il singolar fenomeno che le ceppaie degli Abeti talvolta presentano dopo l'abbatti mento dei loro fusti, consistente nella successiva produzio ne per più anui consecutivi di uno strato legnoso all'anno, fenomeno che ha formato per molto tempo un argomento validissimo contro la teoria del Dupetit-Thouars. Al presento si può dire che per le ricerche del Sig. H. R. Goeppert la Scienza possiede una completa descrizione, e spiegazione di tal fenomeno. Per le osservazioni che il medesimo fece nel 1840 su tal proposito in una foresta della Slesia, ricca di Abeti, potè accertarsi che ogni qual volta tal fenomeno'arveniva, vi era sempre nelle ceppaie trasfusione di nutrimento dagli Abeti vegetanti in prossimità, trasfusione operata per piu o meno completo saldamento delle radici .

Le osservazioni sono le seguenti.

Dopo che il tronco è stato abbattuto, il nuovo strato legnoso si organizza fra la scorza ed il legno in tutta l' estensione delle radici, e dalla base della ceppaia procede verso 